\title{
Dural Sinus Malformation with Dural Arteriovenous Fistula
}

\author{
C. KWONG YAU, H. ALVAREZ*, P. LASJAUNIAS* \\ Department of Neurosurgery, Kwong Wah Hospital, Hong Kong \\ * Department of Neuroradiology, Bicetre Hospital, Le Kremlin Bicetre, France
}

Key words: dural sinus malformation, dural AV shunt, single hole fistula, transarterial embolization, infancy

\section{Summary}

A rare case of dural sinus malformation with dural arteriovenous fistula in an infant is presented. Presenting symptom was progressive macrocrania without hydrocephalus. A high flow arteriovenous of the sigmoid sinus associated with jugular bulb diaphragm was demonstrated. Reflux in the intracranial sinus was present. The shunt was successfully occluded by transarterial embolization. The natural history and treatment strategy are discussed.

\section{Introduction}

Pediatric dural arteriovenous (AV) shunt is rare, although it is most often referred to in the literature. Morita et al (1995) had reported a poor prognosis with overall mortality of $38 \%{ }^{1}$.

Three different types of dural AV shunt have been described, namely, the dural sinus malformation with dural $\mathrm{AV}$ shunt, the infantile type and the adult type which differ in their etiology, presentations, and prognosis ${ }^{2}$.

\section{Clinical History}

The patient was a 15-month-old boy who presented with progressive macrocrania for several months. In addition, the parents found that there was a pulsatile right retroauricular mass. Physical examination showed a wellnourished child with mild macrocrania. There was loco-regional dilation of the vessels with bruit at the retroauricular region. The anterior fontanelle was soft. Clinically, there was no tachycardia and there were no signs of heart failure. Developmentally, he had normal milestones.

Doppler examination was performed which found a fistula connecting the retromastoid branch of the right occipital artery and the transverse sinus.

MRI of the brain showed that there was dilated scalp vessel at the right retroauricular region. The right distal sigmoid sinus was absent. There was normal brain parenchyma and no hydrocephalus was found. There was no tonsillar herniation. MRA showed an enlarged right occipital artery. The ipsilateral jugular vein was present.

\section{Angiogram and Embolization}

The right occipital artery was dilated and tortuous on external carotid injection. A high flow fistula was found connecting the occipital artery to the transverse-sigmoid junction. (figure 1).

The right distal sigmoid sinus could not be visualized. Venous drainage of the AVF was mainly to the opposite internal jugular vein through the torcular. Persistent and enlarged occipital sinus was also found which drained to the ipsilateral internal jugular vein through the 


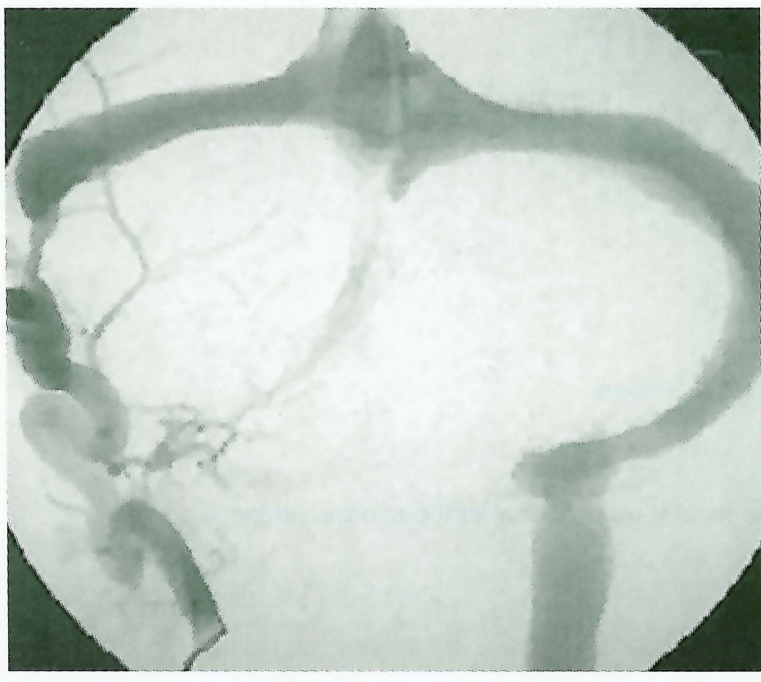

Figure 1

marginal sinus. Selective internal maxillary artery injection showed both the petrous and squamous branches of the middle meningeal artery provided feeders that converged to the same fistulous site (figure 2).

The right internal carotid did not have any feeders to the fistula (figure 3 ). In the venous phase, in contrast to the external carotid angiogram, the right transverse sinus could not be visualized. The right hemisphere drained through the opposite transverse sinus to the opposite internal jugular vein or to ipsilateral internal jugular vein through the occipital sinus. (figure 4).

No cortical venous reflux was demonstrated. In the vertebral injections it showed the artery

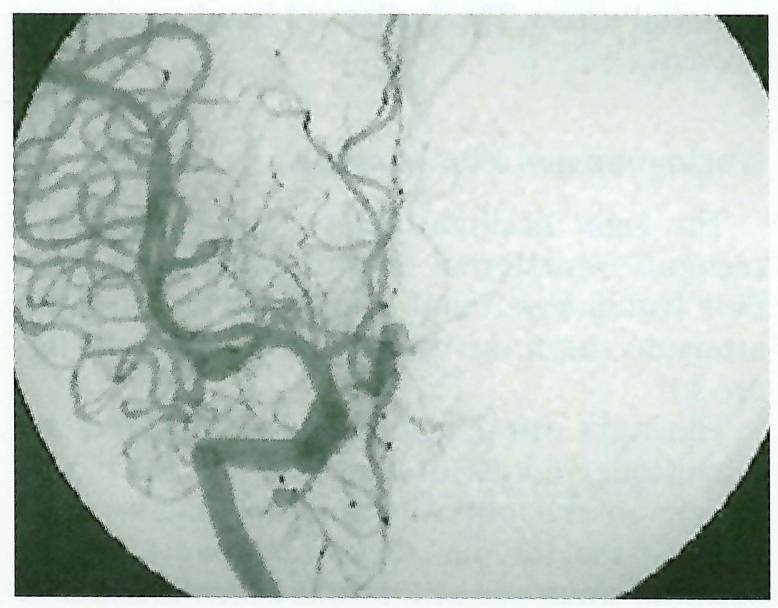

Figure 3

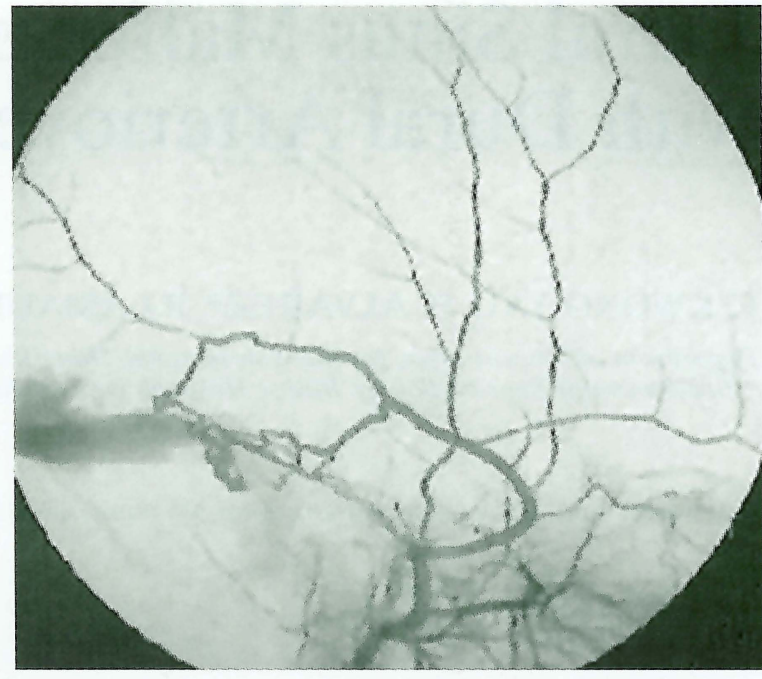

Figure 2

of the falx cerebellum supply the dural fistula across the midline.

The right occipital artery was then catheterized with a microcatheter (Sense CP, Cath Net System Paris, France) and was navigated to the fistulous site. The fistula was embolized with a single injection of undiluted NBCA glue with tantalum powder (figure 5).

The post-embolization angiogram showed complete obliteration of the fistula. The right transverse sinus became visible on the internal carotid injection because of the diminished flow from the fistula. The supply from the right middle meningeal artery, and dural branch of the vertebral artery no longer opacified the shunt (figure 6).

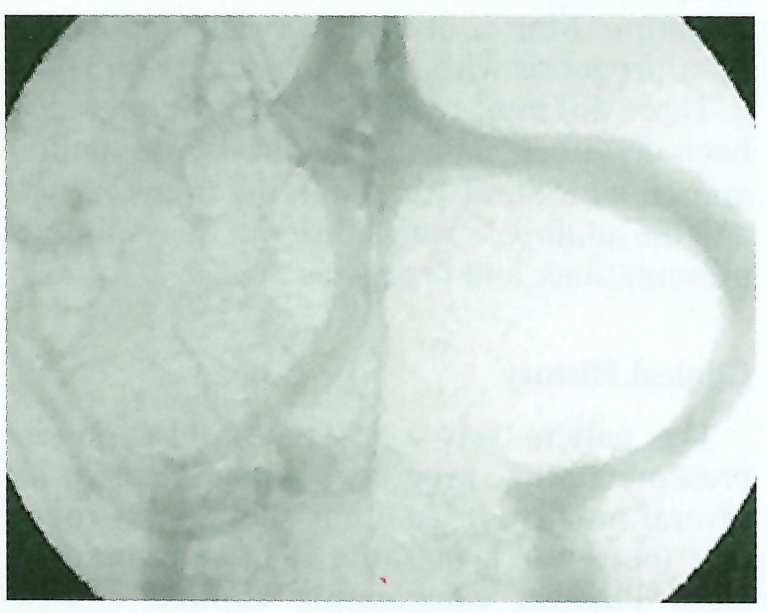

Figure 4 


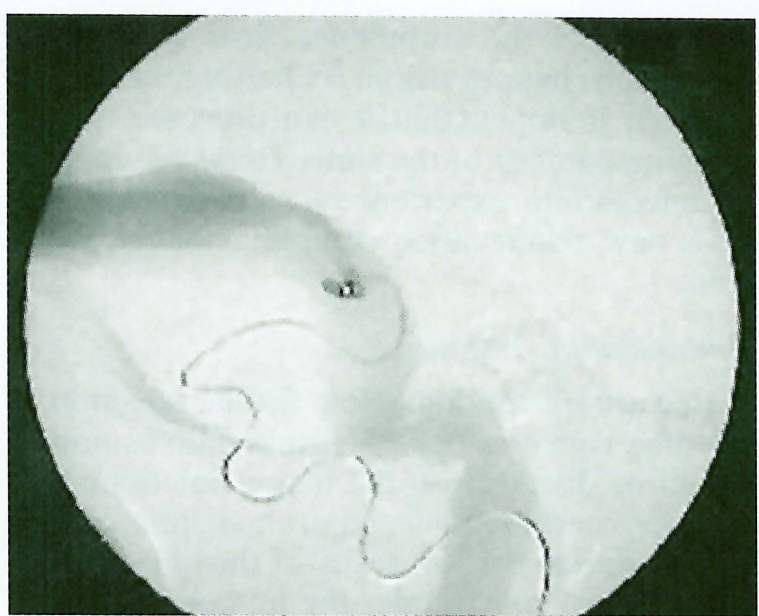

Figure 5

\section{Discussion}

\section{Dural Sinus Malformation}

It is worth noting that although the sigmoid and the jugular sinuses have already formed at the $80 \mathrm{~mm}$ stage, the expansion and maturation of the sigmoid-jugular junction and the formation of the superior jugular bulb is a post-natal event up to two years of age $e^{2,3,4,5}$.

Most cases of dural sinus malformation are, by definition, associated with the absence or maldevelopment of the posterior sinuses including transverse or sigmoid sinus. Cases with persistent sigmoid diaphragm have also been previously demonstrated ${ }^{2}$. In our patient, there was persistence of the enlarged occipital sinus which drained into the inferior end of the sigmoid sinus through the marginal sinus, adopting the fetal configuration. The development of the dural AVS is likely to be secondary to malformation of the sinus.

\section{Histopathogy \& Pathogenesis}

The classic dural AV fistulas are located in the dura near venous sinuses and within the sinus walls. However, no direct fistula between the dural artery and venous sinus is found histologically. Instead of capillaries, the dural arteries communicate with the dural vein through intervening small vessels which have one layer of endothelium and an incomplete smooth muscle layer $6,7,8$.

The exact etiology is still controversial. In contrast to adults, dural AVFs in infant or pedi-

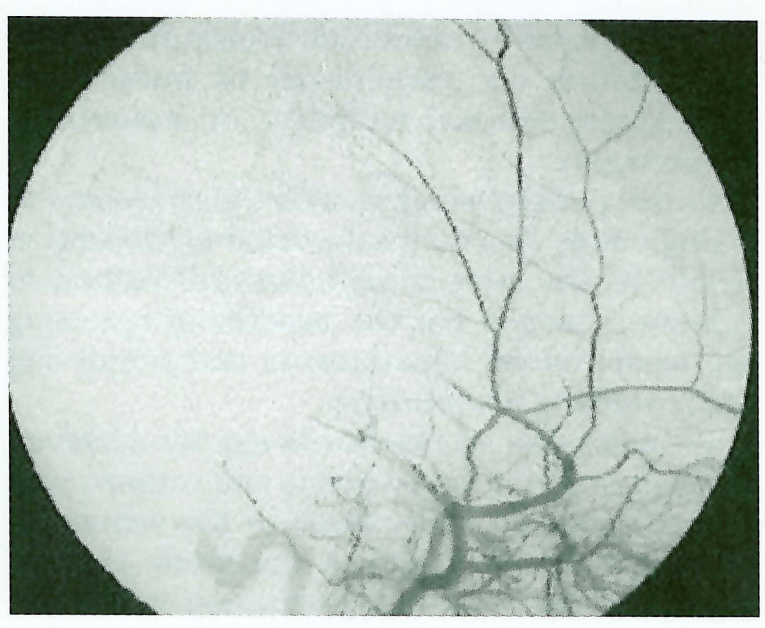

Figure 6

atric patients are assumed to be congenital. The most widely accepted cause is the maldevelopment or stenosis of the sinuses. Venous stenosis is the primary pathophysiological mechanism responsible for venous hypertension ${ }^{9}$. On the other hand, in response to the raised venous pressure, there would be arterialization of the vein with intimal thickening, collagen deposition, and smooth muscle hyperplasia. Arterialization of the veins further reduces the lumen and increases venous pressure ${ }^{6,9}$. A self-propagating vicious cycle is then set up. Furthermore, endothelial properties of the malformed sinuses also change with a high propensity of spontaneous thrombosis. This would result in complete occlusion of all the venous outlets and acute clinical deterioration.

The presence of the jugular bulb diaphragm is unexplained but is observed as the consequence of the dysmaturation of the posterior sinuses during infancy. Triggers of such event include high flow shunt like in Vein of Galen aneurysmal malformation, or craniostenosis.

\section{Natural History and Goal of Treatment}

With the concept of pathogenesis and natural history in mind, the treatment principle is to correct the venous hypertension by interrupting the AV fistula and preserving the remaining patent sinus ${ }^{2}$ (figure 7). Bruit is not a symptom at that age, as the baby considers the pulsatile bruit a normal "noise". Its disappearance after complete cure may disorientate the child for a few days and should be carefully considered.

Fistulas draining into the venous sinus have 
been managed by neuroradiologists either with embolization via transarterial or transvenous routes, conventional radiation, or stereotactic radiosurgery.

In this patient who has converging feeders to a single hole fistula, transarterial embolization would be the most straightforward method to achieve a cure. It has the benefits of reversing the local venous hypertension and preserving the remaining patent sinus.

Embolization of the fistula can be achieved through both the transvenous and transarterial routes. Although obliteration of the venous sinus can secondarily occlude the fistula, the resolution of the venous hypertension would depend on the collateral circulation. On the other hand, selective occlusion of the fistula would help control the turbulence flow and reduce the venous hypertension. By halting the vicious circle, it is hoped that it can preserve the remaining patency of the sinus. However, it is not an uncommon experience to see development of a new fistula after complete fistula occlusion.

\section{Embolization Technique}

Because of the high flow fistula, injection of the glue carries a risk of distal embolization. It would be disastrous if the major functioning sinus were occluded instead of the fistula.

In addition, the tortuosity of the enlarged occipital artery may render the microcatheter unstable during the glue injection. Thus, the tip of the microcatheter was optimally positioned at a

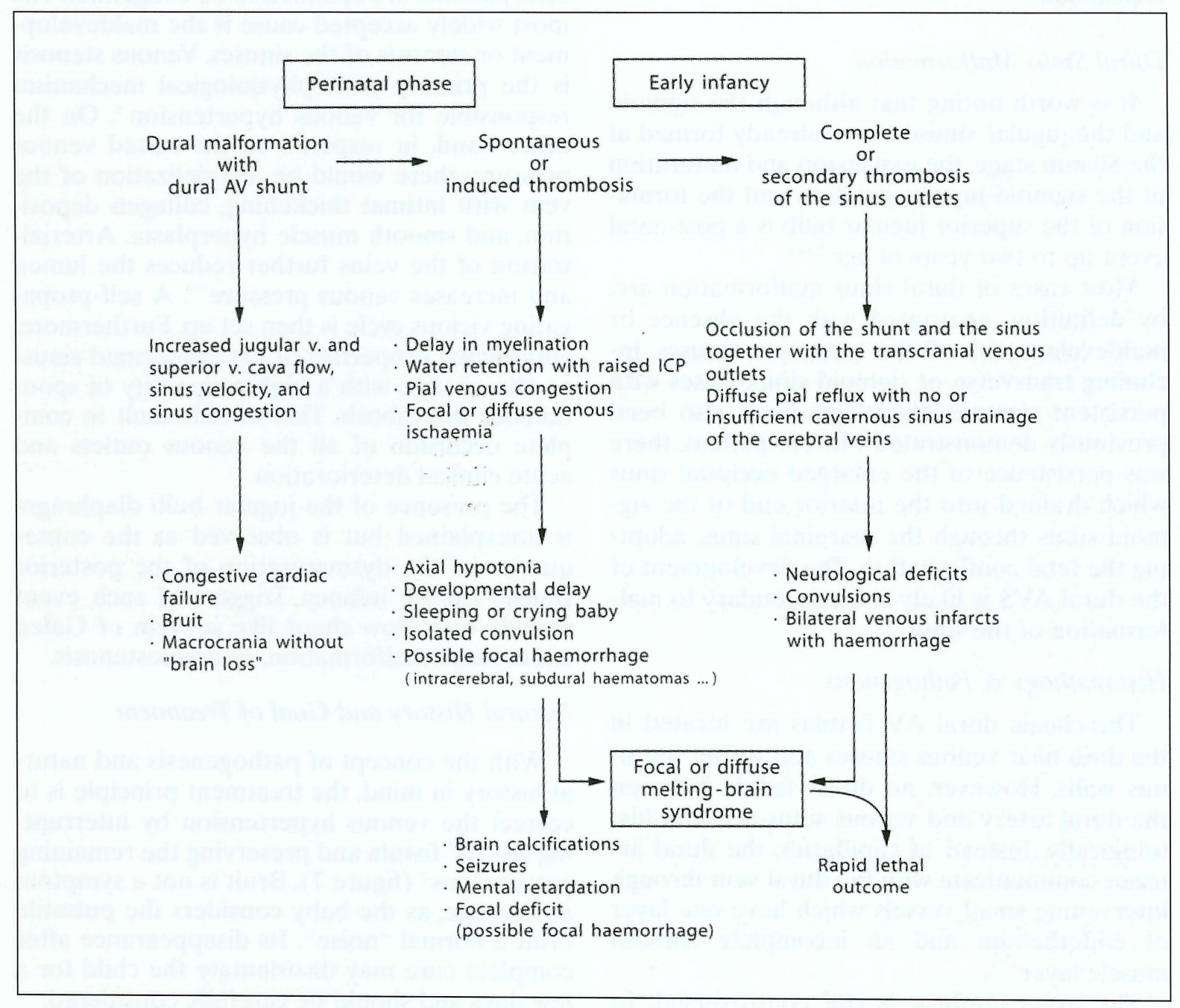

Figure 7 
converging fistulous site pointing at the side wall.

The intention was initially to achieve a good cast of glue formation on the wall of the fistula when it was first out of the catheter tip and secondly to stabilize the catheter during the glue injection.

To augment the glue embolization, a high concentration (near 100\%) of radiopaque (Tantalum powder) glue was used ${ }^{10}$. Furthermore, Valsalva maneuver was used to reduce the flow rate of the fistula during the glue injection.

\section{References}

1 Morita, A, Meyer FB et Al: Childhood dural arteriovenous fistula in the posterior fossa: Three case reports and review of the literature. Neurosurgery 37: 11931200, 1995.

2 Lasjaunias, P: Vascular diseases in neonates, infants, and children. New York: Springer Berlin Heidelberg: 320-371, 1997.

3 Okudera T, Peng HY et Al: 'Developmental radiology of posterior fossa dural sinuses in the human fetus: With special reference to physiological enlargement of transverse and occipital sinuses, formation of emissary veins, and development of superior jugular bulb from jugular sinuses' cited in Hakuba A (ed) Surgery of the intracranial venous system, New York: Springer Berlin Heidelberg, 1996.

4 Miquel MA, Mateu JMD et Al: "Embryogenesis of the veins of the posterior fossa: an overview", cited in Hakuba A (ed) Surgery of the intracranial venous system, New York: Springer Berlin Heidelberg: 14-25, 1996.

5 Ann Gilmore S: Developmental anatomy of the intracranial venous system: Review of the dural venous sinus development ' cited in Hakuba A (ed) Surgery of the intracranial venous system, New York: Springer Berlin Heidelberg: 3-13, 1996.

6 Nishijima M, Takaku, A: 'Clinical problems and histopathological study in patients with transverse-sigmoid sinus dural arteriovenous malformations' cited in Hakuba A (ed) Surgery of the intracranial venous system, New York: Springer Berlin Heidelberg: 425-430, 1996.

\section{Conclusion}

This is a rare case of infantile dural arteriovenous fistula associated with distal sigmoid sinus malformation. There is only a single fistula site and anatomical cure was achieved in one session with a single glue injection. However, there is no single ideal treatment for the obliteration of dural AVFs and the management of each case is best considered individually. Follow-up vascular study is required to assess the status of the venous drainage and to rule out the recurrence of the fistula.
7 Nakamura M, Tamaki $\mathrm{N}$ et $\mathrm{Al}$ : Two unusual cases of multiple dural arteriovenous fistula. Neurosurgery 41 : 288-293, 1997.

8 Yasuhiro H, Katsuya $\mathrm{G}$ et Al: Histopathological aspects of the dural arteriovenous fistula in the transverse sigmoid sinus region in 9 patients. Neurosurgery 40: 452-458, 1997

9 Tew JM, Lewis AI: 'Effects of venous hypertension on dural arteriovenous malformations' cited in Hakuba A (ed) Surgery of the intracranial venous system. New York: Springer Berlin Heidelberg: 14-25, 1996.

10 Berenstein A, Lasjaunias P: Surgical Neuroangiography, New York: Springer Berlin Heidelberg (4), 1993.
Chan Kwong Yau, M.D.

Department of Neurosurgery Kwong Wah Hospital, Hong Kong e-mail: kwongyauchan@netscape.net 